\title{
Relating Edelman-Greene insertion to the Little map
}

\author{
Zachary Hamaker • Benjamin Young
}

Published online: 1 April 2014

(C) Springer Science+Business Media New York 2014

\begin{abstract}
The Little map and the Edelman-Greene insertion algorithm, a generalization of the Robinson-Schensted correspondence, are both used for enumerating the reduced decompositions of an element of the symmetric group. We show the Little map factors through Edelman-Greene insertion and establish new results about each map as a consequence. In particular, we resolve some conjectures of Lam and Little.
\end{abstract}

Keywords Reduced words · Little map $\cdot$ Edelman-Greene $\cdot$ Stanley symmetric functions $\cdot$ Dual equivalence $\cdot$ Sorting network $\cdot$ Coxeter-Knuth move .

Robinson-Schensted algorithm

\section{Introduction}

\subsection{Preliminaries}

In this paper, we clarify the relationship between two algorithmic bijections, due to Edelman and Greene [6] and to Little [14], respectively, both of which deal with reduced decompositions in the symmetric group $S_{n}$. It is well known that $S_{n}$ can be viewed as a Coxeter group with the presentation

$S_{n}=\left\langle s_{1}, s_{2}, \ldots, s_{n-1}\right| s_{i}^{2}=1, s_{i} s_{j}=s_{j} s_{i}$ for $\left.|i-j| \geq 2, s_{i} s_{i+1} s_{i}=s_{i+1} s_{i} s_{i+1}\right\rangle$

\footnotetext{
Z. Hamaker

Department of Mathematics, Dartmouth College, 27 N. Main Street, 6188 Kemeny Hall, Hanover, NH 03755-3551, USA

e-mail: zachary.hamaker@gmail.com

B. Young $(\bowtie)$

Department of Mathematics, 1222 University of Oregon, Eugene, OR 97403-1205, USA

e-mail: bjy@uoregon.edu
} 
where $s_{i}$ can be viewed as the transposition $(i i+1)$. Let $\sigma=\sigma_{1}, \sigma_{2}, \ldots, \sigma_{n} \in S_{n}$. A reduced decomposition or reduced expression of $\sigma$ is a minimal-length sequence $s_{w_{1}}, s_{w_{2}}, \ldots, s_{w_{m}}$ such that $\sigma=s_{w_{1}}, s_{w_{2}}, \ldots, s_{w_{m}}$. The word $w=w_{1}, w_{2}, \ldots, w_{m}$ is called a reduced word of $\sigma$. It is convenient to refer to a reduced decomposition by its corresponding reduced word and we will conflate the two often. The set of all reduced decompositions of $\sigma$ is denoted $\operatorname{Red}(\sigma)$. An inversion in $\sigma$ is a pair $(i, j)$ with $i<j$ and $\sigma_{i}>\sigma_{j}$. Let $l(\sigma)$ be the number of inversions in $\sigma$. Since each transposition $s_{i}$ either introduces or removes an inversion, for $w=w_{1}, \ldots, w_{m}$ a reduced word of $\sigma$, we can show $m=l(\sigma)$.

The enumerative theory of reduced decompositions was first studied in [18], where using algebraic techniques it is shown for the reverse permutation $\sigma=n \ldots 21$ that

$$
|\operatorname{Red}(\sigma)|=\frac{\left(\begin{array}{l}
n \\
2
\end{array}\right) !}{(2 n-3)(2 n-5)^{2} \cdots 5^{n-2} 3^{n-2}} .
$$

This is the same as the number of standard Young tableaux with the staircase shape $\lambda=$ $(n-1, n-2, \ldots, 1)$. In addition, Stanley conjectured for arbitrary $\sigma \in S_{n}$ that $|\operatorname{Red}(\sigma)|$ can be expressed as the number of standard Young tableaux of various shapes (possibly with multiplicity). This conjecture was resolved in [6] using a generalization of the Robinson-Schensted insertion algorithm, usually called Edelman-Greene insertion. Edelman-Greene insertion maps a reduced word $w$ to the pair of Young tableaux $(P(w), Q(w))$ where the entries of $P(w)$ are row-and-column strict and $Q(w)$ is a standard Young tableau. The same map also provides a bijective proof of (1), as there is only one possibility for $P(w)$ while every standard $Q(w)$ is possible.

Algebraic techniques developed in [11] can be used to compute the exact multiplicity of each shape for given $\sigma$. A bijective realization of Lascoux and Schützenberger's techniques in this setting is demonstrated in [14]. A descent is an inversion of the form $(i, i+1)$. Permutations with precisely one descent are referred to as Grassmannian. There is a simple bijection between reduced words of a Grassmannian permutation $\sigma$ and standard Young tableaux of a shape determined by $\sigma$. The Little map works by applying a sequence of modifications referred to as Little bumps to the reduced word $w$ until the modified word's corresponding permutation is Grassmannian so that it can be mapped to a standard Young tableau denoted $\operatorname{LS}(w)$.

\subsection{Results}

Since the Little map's introduction, there has been speculation on its relationship to Edelman-Greene insertion. In the appendix of [8], written by Little, Conjecture 4.3.2 asserts that $\operatorname{LS}(w)=Q(w)$ when the maps are restricted to reduced words of the reverse permutation. Similar comments are made in [14]. We show that the connection is much stronger than previously suspected: this equality is true for every permutation.

Theorem 1 Let $w$ be a reduced word. Then

$$
Q(w)=L S(w)
$$


The proof is based on an argument from canonical form. First, we verify the theorem for the column reading word, a canonical reduced word associated to $P(w)$ that plays nicely with both Edelman-Greene insertion and Little bumps. We then show the statement's truth is invariant under Coxeter-Knuth moves, transformations that traverse the space of reduced words with identical $P(w)$. Using Theorem 1, we also demonstrate a method for computing the set of $P(w)$ associated to the permutation $\sigma$ that is more efficient than those previously known.

Given Theorem 1, one might suspect the respective structures of the two maps are intimately related. Specifically, Conjecture 2.5 of [10] proposes that Little bumps relate to Edelman-Greene insertion in a way that is analogous to the role dual Knuth transformations play for the Robinson-Schensted-Knuth algorithm.

Let $v$ and $w$ be reduced words. We say $v$ and $w$ communicate if there exists a sequence of Little bumps changing $v$ to $w$. This is an equivalence relation as Little bumps are invertible.

Theorem 2 (Lam's Conjecture) Let $v$ and $w$ be two reduced words. Then $v$ and $w$ communicate if and only if $Q(v)=Q(w)$.

For $\sigma$ a permutation, we define a graph $G_{\sigma}$ on $\operatorname{Red}(\sigma)$ by saying two reduced words are adjacent if they differ by a Coxeter-Knuth move. Using Theorem 2, we demonstrate this graph is a dual equivalence graph, as introduced in [4] and expanded on in [16].

Theorem 3 The graph $G_{\sigma}$ is a dual equivalence graph.

Theorem 3 is an observation of Sara Billey (in a private communication), and our proof strategy follows closely her original proof. A last consequence of our work is Algorithm 4.3, which computes the set of Edelman-Greene $P$ tableaux associated to a permutation. We have not investigated whether this algorithm is more efficient than the algorithms in [15] for the same purpose; however, our algorithm is easier to implement, and perhaps conceptually clearer.

\subsection{Random sorting networks}

In recent years, there has been interest in the properties of randomly chosen reduced decompositions for the reverse permutation, known as random sorting networks. introduced in [3] and studied further in [1,2]. Little is known about these objects rigorously, though conjectures are plentiful, striking, and strongly supported by numerical evidence. Most of the results that are known come from analyzing the asymptotics of staircase-shaped Young tableaux, by way of the Edelman-Greene correspondence.

For instance, it is conjectured in [3, Conjecture 2] that the "partial" permutation matrix of a random sorting network, obtained by concatenating the first half of the transpositions, has its nonzero entries distributed according to the Archimedean distribution. Curiously, this distribution is also found in the limiting shape of a random domino tiling of the Aztec Diamond [5]. However, the current best result in this direction [3, Theorem 4] is an octagonal bound on the support of the nonzero entries in the partial permutation matrix. The bound is obtained by computing the limiting profile of 
a random staircase Young tableau (which can be done precisely), and then attempting to push this information through the Edelman-Greene correspondence (which cannot).

We think that an incomplete understanding of the Edelman-Greene algorithm is one of the main obstacles to progress on the random sorting network problem. As such, we hope that by strengthening the combinatorial foundations of this area, better asymptotic characterizations of random sorting networks will be attained. We regard this paper as a first step in this direction.

\subsection{Structure of the paper}

In the second section, we review those parts of $[6,14]$ which we need: we define Edelman-Greene insertion and the Little map, as well as generalized Little bumps. Additionally, we state some properties of these maps that are important to our work. The third section defines Coxeter-Knuth transformations and studies their interaction with Little bumps and action on $Q(w)$. We conclude in the fourth section by proving our main results and resolving several conjectures of Little and Lam.

\section{Two maps}

\subsection{Edelman-Greene insertion}

In order to define Edelman-Greene insertion, we must first define a rule for inserting a number into a tableau. Let $n \in \mathbb{N}$ and $T$ be a tableau with rows $R_{1}, R_{2}, \ldots, R_{k}$ where $R_{i}=r_{1}^{i} \leq r_{2}^{i} \leq \cdots \leq r_{l_{i}}^{i}$. We define the insertion rule for Edelman-Greene insertion, following [6].

1. If $n \geq r_{l_{1}}^{1}$ or if $R_{1}$ is empty, adjoin $n$ to the end of $R_{1}$.

2. If $n<r_{l_{1}}^{1}$, let $j$ be the smallest number such that $n<r_{j}^{1}$.

(a) If $r_{j}^{1}=n+1$ and $r_{j-1}^{1}=n$, insert $n+1$ into $T^{\prime}=R_{2}, \ldots, R_{k}$ and leave $R_{1}$ unchanged.

(b) Otherwise, replace $r_{j}^{1}$ with $n$ and insert it into $T^{\prime}=R_{2}, \ldots, R_{k}$.

Aside from 2(a), this is the RSK insertion rule. Such exceptional bumps are referred to as special. For $w=w_{1} \ldots w_{m}$ a word (not necessarily reduced), we define $\operatorname{EG}(w)=$ $(P(w), Q(w))$ via the following sequence of tableaux (see Fig. 1 for an example). We obtain $P_{1}(w)$ by inserting $w_{m}$ into the empty tableau. Then $P_{j}(w)$ is obtained by inserting $w_{m-j+1}$ into $P_{j-1}(w)$. Note we are inserting the entries of $w$ from right to left. At each step, one additional box is added. In $Q(w)$, the entry of each box records the time of the step in which it was added. From this, we can conclude that $Q(w)$ is a standard Young tableau. Note the fourth insertion in Fig. 1 follows 2(a). For $w$ a reduced word of some $\sigma$, it is shown in [6] that the entries of $P(w)$ are strictly increasing across rows and down columns. Additionally, we can recover $\sigma$ from $P(w)$ with no additional information, that is $P(w)$ determines $\sigma$. 


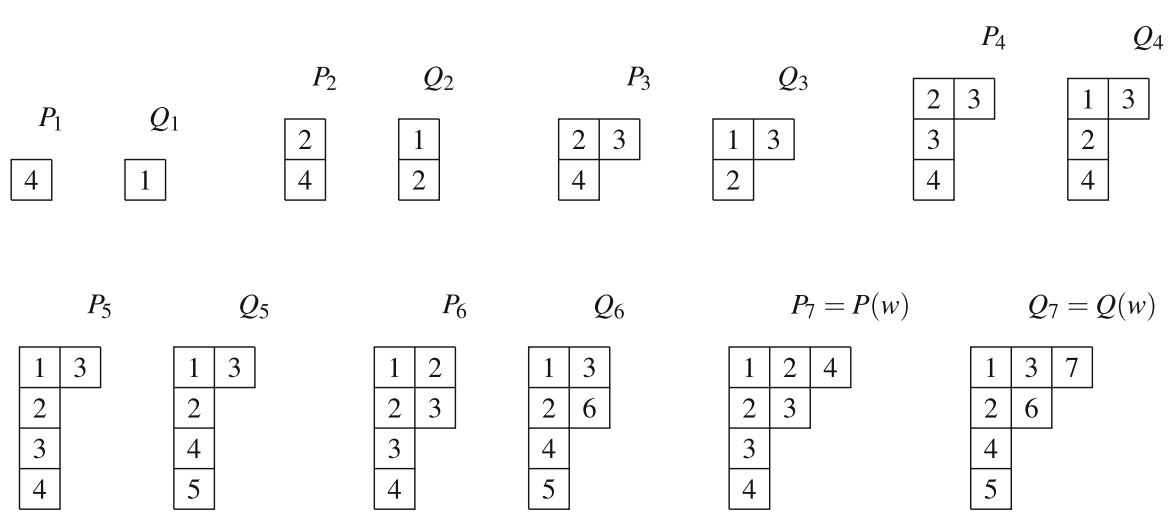

Fig. 1 Edelman-Greene insertion for $w=4,2,1,2,3,2,4$

\subsection{Grassmannian permutations}

Recall a permutation $\sigma$ is Grassmannian if it has exactly one descent. We can then write

$$
\sigma=a_{1} a_{2} \ldots a_{k} b_{1} b_{2} \ldots b_{n-k}
$$

where $\left\{a_{i}\right\}_{i=1}^{k}$ and $\left\{b_{j}\right\}_{j=1}^{n-k}$ are increasing sequences with $a_{k}>b_{1}$. A word $w$ is Grassmannian if it is the reduced word of a Grassmannian permutation. From the Grassmannian word $w=w_{1}, \ldots, w_{m}$ we construct a tableau Tab $(w)$ as follows. Index the columns of $\operatorname{Tab}(w)$ by $b_{1}, \ldots, b_{n-k}$ and the rows by $a_{k}, a_{k-1}, \ldots, a_{1}$. Since all inversions in $\sigma$ feature an $a_{i}$ and a $b_{j}$, each $w_{l}$ in $w$ represents the swap between an $a_{i}$ and a $b_{j}$. For $w_{l}$, we enter $m+1-l$ in the column indexed by $a_{i}$ and $b_{j}$. If $a_{i}$ swaps with $b_{j}$, we see it must later swap with each smaller $b$. This shows entries are increasing across rows. Likewise, if $b_{j}$ swaps with $a_{i}$, it must later swap with each larger $a$ so entries increase down columns. From this, we can conclude that $\operatorname{Tab}(w)$ is a standard Young tableau whose shape is determined by $\sigma$. There is an example of Tab in Fig. 2. For a given Grassmannian permutation $\sigma$, this map is a bijection as the process is easily reversed. Multiple Grassmannian permutations may correspond to the same shape. However, they will only differ by some fixed points at the beginning and end of the permutation.

\subsection{Little bumps and the Little map}

We now describe the method in [14] for transforming an arbitrary reduced word into the reduced word of a Grassmannian permutation. This method is implemented in an easy-to-use Java applet [13] and we strongly recommend that the reader follow along in our descriptions using this software, if possible. Let $w=w_{1} \ldots w_{m}$ be a reduced word and $w^{(i)}=w_{1} \ldots w_{i-1} w_{i+1} \ldots w_{m}$. We construct

$$
w^{(i-)}= \begin{cases}w_{1} \ldots w_{i-1}\left(w_{i}-1\right) w_{i+1} \ldots w_{m} & \text { if } w_{i}>1 \\ \left(w_{1}+1\right) \ldots\left(w_{i-1}+1\right) w_{i}\left(w_{i+1}+1\right) \ldots\left(w_{m}+1\right) & \text { if } w_{i}=1\end{cases}
$$



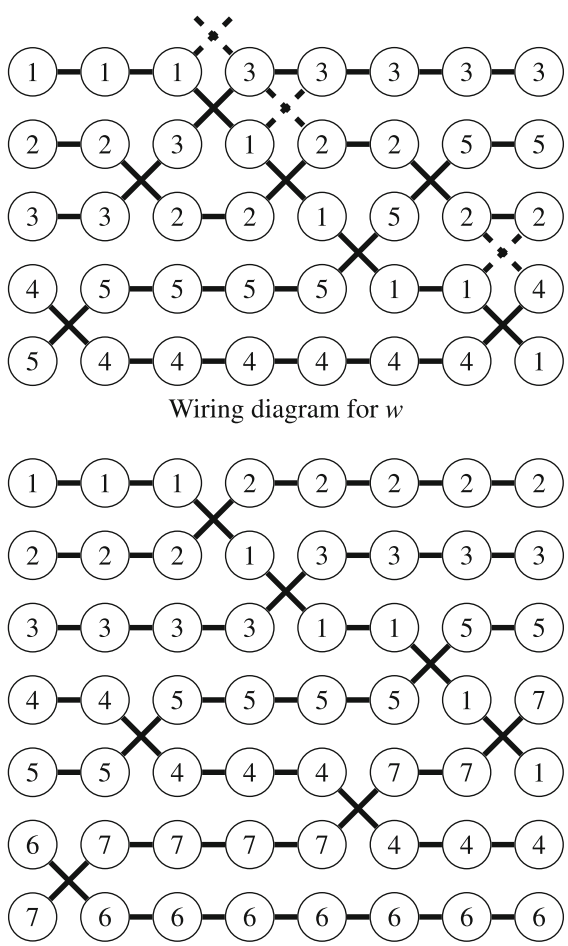

Wiring diagram for $w \uparrow_{7} \uparrow_{7}$

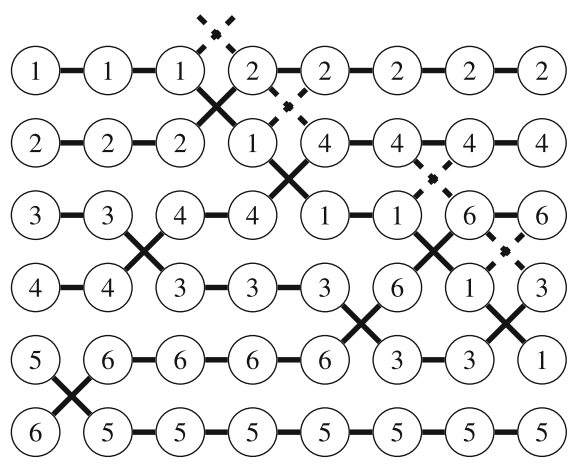

Wiring diagram for $w \uparrow 7$

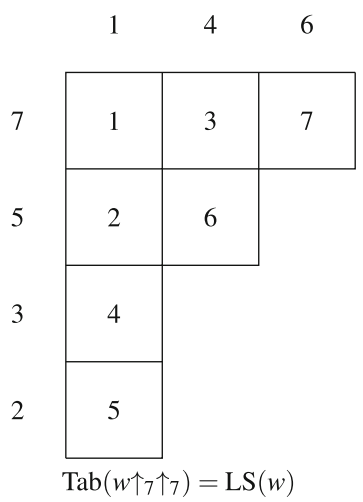

Fig. 2 The Little map for the reduced word $w=4212324$ of $\sigma=35241$. The dashed crosses show the modifications made by the next Little bump

by decrementing $w_{i}$ by one or incrementing each other entry if $w_{i}=1$.

Let $w$ be a reduced word such that $w^{(i)}$ is also reduced. Note $w^{(i-)}$ may not be reduced, as $w_{i}-1$ may swap the same values as some $w_{j}$ with $j \neq i$. However, this is the only way $w^{(i-)}$ can fail to be reduced as $w^{(i)}$ is reduced and we have added only one additional swap. Removing $w_{j}$ from $w^{(i-)}$, we obtain a new reduced word $w^{(i-)(j)}$. Repeating this process of decrementation, we can construct $w^{(i-)(j-)}$ and so on until $w$ has been transformed into a reduced word $v=v_{1} \ldots v_{m}$. We refer to this process as a Little bump beginning at position $i$ and say $v=w \uparrow i$, where $i$ is the initial index the bump was started at. To see that this process terminates, we refer to the following lemma.

Lemma 1 (Lemma 5, [14] ) Let $w$ be a reduced word such that $w^{(i)}$ is reduced. Let $i_{1}, i_{2}, \ldots$ be the sequence of indices decremented in $w \uparrow{ }_{i}$. Then no two of $i_{1}, i_{2}, \ldots$ are equal.

Since $w$ is finite, we see the process terminates so that $w \uparrow i$ is well defined. We highlight a property of Little bumps observed in [14], that they preserve the descent structure of $w$. 
Corollary 1 Let $w=w_{1}, \ldots, w_{m}$ and $v=v_{1}, \ldots, v_{m}$ be reduced words and $\uparrow$ be a Little bump such that $v=w \uparrow$. Then $v_{i}>v_{i+1}$ if and only if $w_{i}>w_{i+1}$ for all $i$.

Proof Let $w_{i}>w_{i+1}$. As each $w_{i}$ is decremented at most once, we see $v_{i} \geq v_{i+1}$, but $v_{i} \neq v_{i+1}$. Thus, $v_{i}>v_{i+1}$. By the same reasoning, if $w_{i}<w_{i+1}$, we see $v_{i}<v_{i+1}$.

Let $w$ be a reduced word of $\sigma \in S_{n}$. We define the Little map LS $(w)$.

1. If $w$ is a Grassmannian word, then $\operatorname{LS}(w)=\operatorname{Tab}(w)$

2. If $w$ is not a Grassmannian word, identify the swap location $i$ of the last inversion (lexicographically) in $\sigma$ and output $\operatorname{LS}(w \uparrow i)$.

It is a result from [14] extending work in [11] that LS terminates. We then see that $w \mapsto \operatorname{LS}(w)$ where $\operatorname{LS}(w)$ is a standard Young tableau. An example can be seen in Fig. 2, where the word $w$ is represented by its wiring diagram: an arrangement of horizontal, parallel wires spaced one unit apart, labeled 1 through $n$ on the left-hand side, in which each letter in the word $w$ is represented by a crossing of wires.

\section{The action of Coxeter-Knuth moves}

\subsection{Basics of Coxeter-Knuth moves}

First introduced in [6], Coxeter-Knuth moves are perhaps the most important tool for studying Edelman-Greene insertion. They are modifications of the second and third Coxeter relations. Let $a<b<c$ and $x$ be integers. The three Coxeter-Knuth moves are the modifications

1. $a c b \leftrightarrow c a b$

2. $b a c \leftrightarrow b c a$

3. $x(x+1) x \leftrightarrow(x+1) x(x+1)$

applied to three consecutive entries of a reduced word. Let $w=w_{1}, w_{2}, \ldots, w_{m}$ be a reduced word of $\sigma$ and $\alpha$ denote a Coxeter-Knuth move on the entries $w_{i-1} w_{i} w_{i+1}$. Since $a<b<c$, if $\alpha$ is of type one or two we have $w \alpha$ a reduced word of $\sigma$ as well by the second Coxeter relation. If $\alpha$ is of type three then $w \alpha$ is a reduced word of $\sigma$ by the third Coxeter relation. We say two reduced words $v$ and $w$ are Coxeter-Knuth equivalent if there exists a sequence $\alpha_{1}, \alpha_{2}, \ldots, \alpha_{k}$ of Coxeter-Knuth moves such that

$$
v=w \alpha_{1} \ldots \alpha_{k}
$$

Note that two Coxeter-Knuth equivalent reduced words must correspond to reduced decompositions of the same permutation. We can see their action on wiring diagrams in Fig. 3.

Coxeter-Knuth moves play a role in the study of Edelman-Greene insertion analogous to that of Knuth moves in the study of RSK insertion.

Theorem 4 (Theorem 6.24 in [6]) Let $v$ and $w$ be reduced words. Then $P(v)=P(w)$ if and only if $v$ and $w$ are Coxeter-Knuth equivalent. 


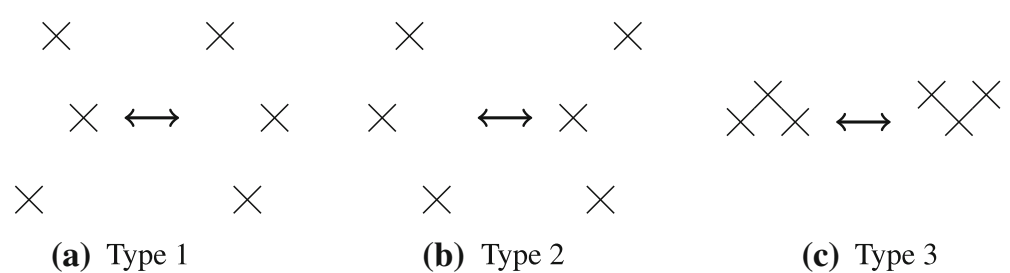

Fig. 3 The three types of Coxeter-Knuth moves acting on wiring diagrams

\subsection{The action of Coxeter-Knuth moves on $Q(w)$}

In order to understand the relationships of Coxeter-Knuth moves and Little bumps, we must first understand in greater detail how Coxeter-Knuth moves relate to EdelmanGreene insertion. From Theorem 4, we understand how Coxeter-Knuth moves relate to $P(w)$. We must also understand their action on $Q(w)$. For $T$ a standard Young tableau with $n$ entries, let $T t_{i, j}$ be the Young tableau obtained by swapping the entries labeled $n-i$ and $n-j$. Note this notation is unintuitive, as we use $i$ to index the position in the word, rather than the label in $T$.

Lemma 2 Let $w=w_{1} \ldots w_{m}$ be a reduced word and $\alpha$ be a Coxeter-Knuth move on $w_{i-1} w_{i} w_{i+1}$. If $\alpha$ is a Coxeter-Knuth move of type one or three, then

$$
Q(w \alpha)=Q(w) t_{i-1, i}
$$

If $\alpha$ is a Coxeter-Knuth move of type two, then $\alpha$ acts on $Q(w)$ as above or

$$
Q(w \alpha)=Q(w) t_{i, i+1}
$$

Proof For $w=w_{1} \ldots w_{m}$ a reduced word we see $w_{i-1}:=w_{i-1} w_{i} \ldots w_{m}$ is also a reduced word. Let $\alpha$ be a Coxeter-Knuth move on $w_{i-1} w_{i} w_{i+1}$. By Theorem ?? we see

$$
P\left(\left.w\right|_{i-1}\right)=P\left(\left.w\right|_{i-1} \alpha\right)=P\left(\left.w \alpha\right|_{i-1}\right)
$$

as they differ by a Coxeter-Knuth move. Since $w_{1} \ldots w_{i-2}$ remain unmodified, their insertion is unchanged. Additionally, as $\left.w\right|_{i+2}=\left.w \alpha\right|_{i+2}$ we see $Q\left(\left.w\right|_{i+2}\right)=$ $Q\left(\left.w \alpha\right|_{i+2}\right)$, so changes in $Q(w)$ can only occur at the entries labeled $m-i+1, m-i$ and $m-i-1$. The remainder of this argument is adapted from the proof for Theorem 6.24 in [6]. The strategy of proof is to analyze the insertion of the triplet $w_{i-1} w_{i} w_{i+1}$ and its counterpart in $w \alpha$ into one row of $P\left(\left.w\right|_{i+2}\right)$. If one such $w_{j}$ fails to bump anything, the analysis is straightforward. Otherwise the three entries bumped by each will continue to differ by a Coxeter-Knuth move, allowing us to reduce the problem to the previous case.

1. In this case, we do not need to complete the full analysis described above. Let $\alpha$ be a Coxeter-Knuth move of type one. Then $w_{i+1}$ inserts into the same spot in $P\left(\left.w\right|_{i+2}\right)$ for both $w$ and $w \alpha$. Since $Q(w) \neq Q(w \alpha)$, we see $Q(w \alpha)=Q(w) t_{i-1, i}$. 
2. Let $\alpha$ be a Coxeter-Knuth move of type three. This case is treated now as we will use it in the case where $\alpha$ is a move of type two. We compare the insertion of $x(x+1) x$ and $(x+1) x(x+1)$ into the same row of $P\left(\left.w\right|_{i+2}\right)$. Assume both $x$ and $x+1$ bump an entry of the row. Let $p$ denote the entry bumped by $x, \epsilon_{1}$ be the entry preceding $p$ and $\epsilon_{2}$ be the entry following $p$. If $p>x+1$, we see $x$ and $x+1$ are inserted into the same position, so $Q(w \alpha)=Q(w) t_{i-1, i}$ as in the first case. Let $p=x+1$. Since $\left.w\right|_{i+2}$ is reduced, $\epsilon_{2}=x+2$ (otherwise, inserting $x+1$ first would leave consecutive occurrences of $x+1)$. There are two remaining possibilities: $\epsilon_{1}<x$ or $\epsilon_{1}=x$. Let $\epsilon_{1}<x$. Upon inserting $x(x+1) x$ into the row, we see the first $x$ bumps $x+1, x+1$ bumps $x+2$ and the second $x$ bumps the $x+1$ just inserted (a special bump), so that $(x+1)(x+2)(x+1)$ is inserted into the next row. Upon inserting $(x+1) x(x+1)$ into the row, we see the first $x+1$ produces a special bump of $x+2$, the $x$ bumps $x+1$ and the second $x+1$ bumps the $x+2$ remaining after the special bump, so that $(x+2)(x+1)(x+2)$ is inserted into the next row. The case where $\epsilon_{1}=x$ is simpler. Every bump is a special bump, so that $p$ and $\epsilon_{2}$ are unchanged throughout the insertion process. Each $x$ and $x+1$ will bump an entry precisely one larger, so that the entries to be inserted into the next row will be $(x+1)(x+2)(x+1)$ and $(x+2)(x+1)(x+2)$, respectively. In both cases, we are left with a Coxeter-Knuth move of type three.

If one of the three inserted letters does not bump an entry of the row, we see the largest entry $k$ of the row must be less than $x+1$. As $P\left(\left.w \alpha\right|_{i+1}\right)$ is row and column strict, we see $k<x$, so $x$ or $x+1$ would both insert at the end of the row. Thus, $Q(w \alpha)=Q(w) t_{i-1, i}$

3. Let $\alpha$ be a Coxeter-Knuth move of type two. For $a<b<c$, we compare the insertion of $b c a$ and bac into a row of $P\left(\left.w\right|_{i+2}\right)$ bumping $p q r$ and $p^{\prime} q^{\prime} r^{\prime}$, respectively. If $p=p^{\prime}$, we see $Q(w \alpha)=Q(w) t_{i-1, i}$ as as in the first case. Assume $p \neq p^{\prime}$. One can then check that $a$ and $c$ bump the same entries regardless of order, so that $p=q^{\prime}$ and $q=p^{\prime}$. If $b$ bumps the same entry in each case, it is straightforward to see that $p q r$ and $p^{\prime} q^{\prime} r^{\prime}$ differ by a Coxeter-Knuth move of type two. The only way this does not occur is if $q=p+1$ (so they are next to each other) with $c=p$. In this case, upon inserting $b c a$ we obtain $q(q+1) q$, while the insertion of bac produces $(q+1) q(q+1)$ as the bump of $q+1$ by $c$ is special. Therefore, we are left with another Coxeter-Knuth move of type two or one of type three. In the latter case, we see $Q(w \alpha)=Q(w) t_{i-1, i}$ by the second case.

If some letter does not bump an entry of the row, there are two possibilities. Let $k$ be the largest entry of the row. If $k<a$, then $a$ and $c$ would insert into the same position, so $Q(w \alpha)=Q(w) t_{i-1, i}$. If $a<k<c$, then $c$ inserts on the end of the row and $a$ bumps the same entry $x$ of the row regardless of the order of insertion. Since $x$ is the only entry bumped, we see $P\left(\left.w\right|_{i}\right)=P\left(\left.w \alpha\right|_{i}\right)$. Therefore, $Q(w \alpha)=Q(w) t_{i, i+1}$.

\subsection{Coxeter-Knuth moves and Little bumps}

We now set out to show that Coxeter-Knuth moves commute with Little bumps. This requires two results. The first is that the order we perform a Coxeter-Knuth move $\alpha$ and a Little bump $\uparrow$ does not affect the resulting reduced word. 
Lemma 3 Let $w=w_{1} \ldots w_{m}$ be a reduced word, $\alpha$ a Coxeter-Knuth move on $w_{i-1} w_{i} w_{i+1}$, and $\uparrow_{j, k}$ be a Little bump begun at the swap between the $j$ and $k t h$ trajectories. Then

$$
(w \alpha) \uparrow_{j, k}=\left(w \uparrow_{j, k}\right) \alpha .
$$

Note that $\alpha$ is used here to represent two Coxeter-Knuth moves, possibly of different types, on the same indices.

Proof Let $v=w \uparrow_{j, k}$ and $v^{\prime}=(w \alpha)_{j, k}$. Recall from Lemma 1 and Corollary 1 that $w_{j}-v_{j} \in\{0,1\}$ and $v$ has the same descent structure of $w$.

1. Let $\alpha$ be a Coxeter-Knuth move of the first type, i.e. $w_{i-1} w_{i} w_{i+1} \mapsto w_{i} w_{i-1} w_{i+1}$ with $w_{i+1}$ strictly between $w_{i-1}$ and $w_{i}$. Since a Little bump decrements an entry of $w$ by at most one, one can check that if $w_{i+1}$ differs from $w_{i}$ or $w_{i-1}$ by more than one, we can perform a Coxeter-Knuth move of type one on $v_{i-1} v_{i} v_{i+1}$. In the event that they differ by exactly one and the largest entry is decremented, we see in Fig. 4 that after the bump they differ by a Coxeter-Knuth move of the third type.

2. Let $\alpha$ be a Coxeter-Knuth move of the second type, i.e. $w_{i-1} w_{i} w_{i+1} \mapsto$ $w_{i-1} w_{i+1} w_{i}$ with $w_{i-1}$ strictly between $w_{i+1}$ and $w_{i}$. Since a Little bump decrements an entry of $w$ by at most one, one can check that if $w_{i-1}$ differs from $w_{i}$ or $w_{i+1}$ by more than one, there is a Coxeter-Knuth move of type two on $v_{i-1} v_{i} v_{i+1}$. In the event that they differ by exactly one and the largest entry is bumped, we see in Fig. 4 that after the bump they differ by a Coxeter-Knuth move of the third type.

3. Let $\alpha$ be a Coxeter-Knuth move of the third type. In the event that all three entries are bumped, the resulting entries will continue to differ by a Coxeter-Knuth move of the third type. In the event fewer entries (but not zero) are bumped, we see in Fig. 5 that there will be a Coxeter-Knuth move of the first or second type remaining.
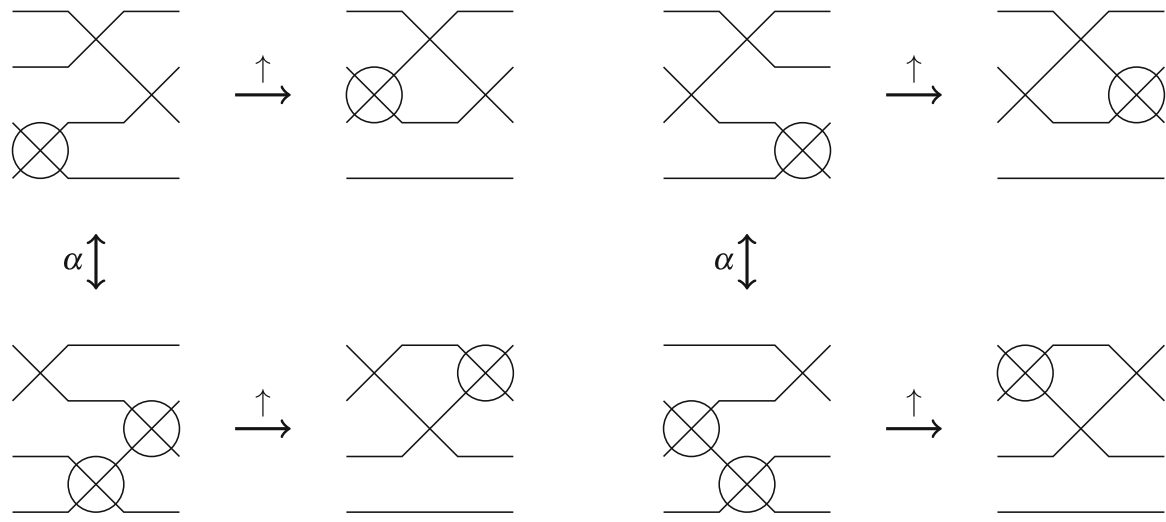

Fig. 4 Transitional bumps for type one and two Coxeter-Knuth moves 


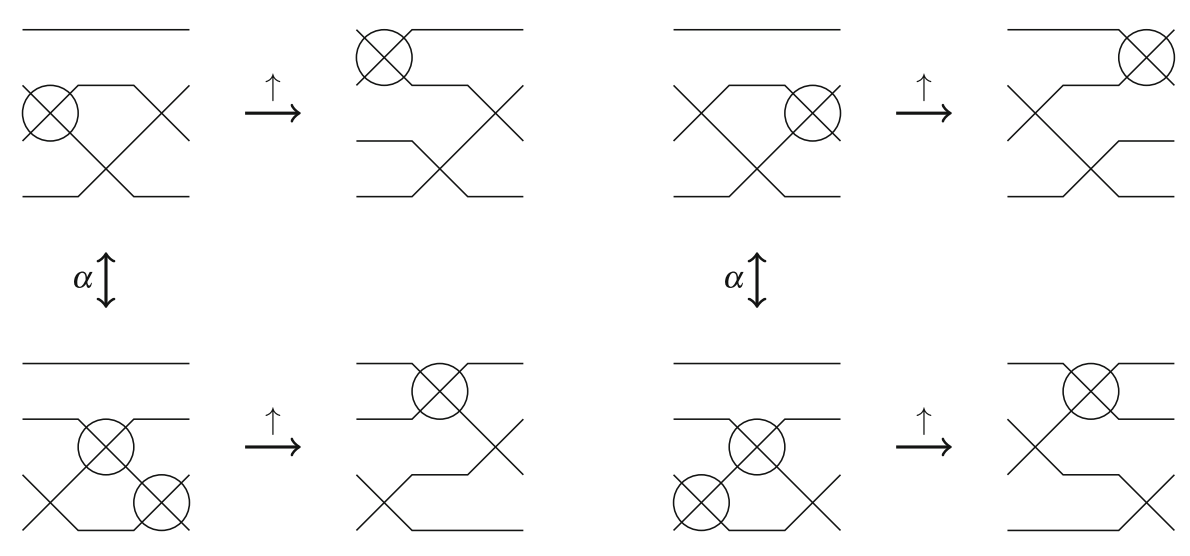

Fig. 5 Transitional bumps for type three Coxeter-Knuth moves

We next show that the rest of the Little bump proceeds in the same manner once the crossings involved in the Coxeter-Knuth move have been bumped. To see this, we need only observe that the last bumped swap is between the same two trajectories. This can be verified readily by examining Figs. 4 and 5 .

The preceding argument assumes that the bumping path does not return to the crossings involved in the Coxeter-Knuth move. It is possible that the bumping path passes through the crossings involved in the Coxeter-Knuth path twice (but no more than that, by Lemma 1). However, the same argument applies, showing that all three crossings are bumped regardless of whether the Coxeter-Knuth move is performed before or after the bump.

We now show that the action of a Coxeter-Knuth move on $Q(w)$ remains the same after applying a Little bump. Combined with Lemma 3, this shows that the order in which Coxeter-Knuth moves and Little bumps are performed on a reduced word $w$ does not affect either the resulting reduced word or the resulting recording tableau.

Lemma 4 Let $w$ be a reduced word, $\alpha$ be a Coxeter-Knuth move and $\uparrow$ a Little bump. Then $Q(w \alpha)=Q(w) t_{i, i+1}$ if and only if $Q(w \uparrow \alpha)=Q(w \uparrow) t_{i, i+1}$.

Proof Let $w=w_{1} \ldots w_{n}$ be a reduced word and $\alpha$ a Coxeter-Knuth move acting on $w$ such that $Q(w \alpha)=Q(w) t_{i, i+1}$. Then by Lemma 2, there are two cases: $\alpha$ is either acting on $w_{i-1} w_{i} w_{i+1}$, or on $w_{i} w_{i+1} w_{i+2}$. We first show $Q((w \alpha) \uparrow)=Q(w \uparrow) t_{i, i+1}$ for the case where $\alpha$ acts on $w_{i-1} w_{i} w_{i+1}$. Note that Lemma 2 implies that $\alpha$ 's action on $w$ is that of a Coxeter-Knuth move of type 2 .

Let $w^{\prime}=w \alpha$. Then $\left.w\right|_{i}=w_{i} w_{i+1} w_{i+2} \ldots w_{n}$ and $\left.w^{\prime}\right|_{i}=w_{i+1} w_{i} w_{i+2} \ldots w_{n}$ are the parts of $w$ and $w^{\prime}$, respectively to the right of $w_{i-1}$. Applying Edelman-Greene insertion to $\left.w\right|_{i}$ and $\left.w^{\prime}\right|_{i}$, we see $Q\left(\left.w\right|_{i}\right)=Q\left(\left.w^{\prime}\right|_{i}\right) t_{i, i+1}$, so $P\left(\left.w\right|_{i}\right)$ and $P\left(\left.w^{\prime}\right|_{i}\right)$ have the same shape. Moreover, we see that $P\left(\left.w\right|_{i-1}\right)=P\left(\left.w^{\prime}\right|_{i-1}\right)$ (that is, after one more insertion the two P-tableaux coincide), since $\left.w\right|_{i-1} \alpha=\left.w^{\prime}\right|_{i-1}$. Additionally, the largest label $n-i-1$ is in the same entry of $Q\left(\left.w\right|_{i-1}\right)$ and $Q\left(\left.w^{\prime}\right|_{i-1}\right)$ so the insertion of $w_{i-1}$ terminates in the same cell. Each step of Edelman-Greene insertion is bijective. Therefore, upon reversing the last insertion, we find $P\left(\left.w\right|_{i}\right)=P\left(\left.w^{\prime}\right|_{i}\right)$. 
Then by Theorem 4, there exists a sequence of Coxeter-Knuth moves $\alpha_{1} \ldots \alpha_{m}$ such that $\left.w\right|_{i}=\left.w^{\prime}\right|_{i} \alpha_{1}, \ldots, \alpha_{m}$. We then see

$$
Q\left(\left.w \uparrow\right|_{i}\right)=Q\left(\left.\left(w^{\prime} \alpha_{1} \ldots \alpha_{m}\right) \uparrow\right|_{i}\right)=Q\left(\left.\left(w^{\prime} \uparrow\right) \alpha_{1} \ldots \alpha_{m}\right|_{i}\right)
$$

by Lemma 3. Therefore, $\left.w \uparrow\right|_{i}$ and $\left.w^{\prime} \uparrow\right|_{i}$ differ solely at their first two positions and are Coxeter-Knuth equivalent, so we see $Q\left(\left.w \uparrow\right|_{i}\right)$ and $Q\left(\left.w^{\prime} \uparrow\right|_{i}\right)$ have the same shape with $Q\left(\left.w \uparrow\right|_{i}\right)=Q\left(\left.w^{\prime} \uparrow\right|_{i}\right) t_{i, i+1}$. Thus, $Q(w \uparrow)=Q\left(w^{\prime} \uparrow\right) t_{i, i+1}=Q(w \alpha \uparrow) t_{i, i+1}$. Since the inverse of a Little bump is also a Little bump, the converse holds as well. Therefore, in the case where $\alpha$ acts on $w_{i-1} w_{i} w_{i+1}$, we see $Q(w \alpha)=Q(w) t_{i, i+1}$ if and only if $Q(w \uparrow \alpha)=Q(w \uparrow) t_{i, i+1}$.

For the case where $\alpha$ acts on $w_{i} w_{i+1} w_{i+2}$, we argue by contradiction. Assume $Q(w \alpha)=Q(w) t_{i, i+1}$, but $Q(w \alpha \uparrow) \neq Q(w \uparrow) t_{i, i+1}$. By Lemma 2 we see $Q(w \alpha \uparrow)=$ $Q(w \uparrow) t_{i+1, i+2}$. Applying the first case to $w \uparrow$, we conclude $Q(w \alpha)=Q(w) t_{i+1, i+2}$, a contradiction. Thus, $Q(w \alpha)=Q(w) t_{i, i+1}$ if and only if $Q(w \uparrow \alpha)=Q(w \uparrow) t_{i, i+1}$.

\section{Proof of results}

\subsection{The Grassmannian case}

Before proving Theorem 1, we need to establish the base case where $w$ is a Grassmannian word. In order to do so, we must understand which entries are exchanging places with each swap. For $w=w_{1} \ldots w_{m}$ a reduced word, we define $\sigma^{i}=s_{w_{1}} s_{w_{2}} \ldots s_{w_{i}}$ where $\sigma^{0}$ is the identity permutation. The $l$ th trajectory of $w$ is the sequence $\left\{\left(\sigma^{i}\right)^{-1}(l)\right\}_{i=0}^{m}$. For $w$ a Grassmannian word of $\sigma=$ $a_{1} a_{2} \ldots a_{k} b_{1} b_{2} \ldots b_{n-k}$, observe that the $j$ th column of $\operatorname{Tab}(w)$ lists the times for all swaps featuring $b_{j}$. Since all such swaps increase the position of $b_{j}$, we can reconstruct its trajectory from the number and location of these swaps. Similarly, we can reconstruct the trajectory of each $a_{i}$ from the $k+1-i$ th row of $\operatorname{Tab}(w)$. We will find it convenient to identify the $l$ th trajectory of a Grassmannian word with the indices $\left\{i_{1}, i_{2}, \ldots, i_{t_{l}}\right\} \subset[n]$ of the swaps featuring $l$. Since insertion takes place from right to left, we index the entries such that $i_{1}>i_{2}>\cdots>i_{t_{l}}$.

Lemma 5 Let $w=w_{1} \ldots w_{m}$ be a reduced decomposition of a Grassmannian permutation $\sigma$. Then $\operatorname{Tab}(w)=Q(w)$.

Proof Let $\sigma=a_{1} a_{2} \ldots a_{k} b_{1} b_{2} \ldots b_{n-k}$ be a Grassmannian permutation with sole descent $a_{k} b_{1}$ and $w=w_{1} \ldots w_{m}$ a reduced decomposition of $\sigma$. Note the trajectories of the $b_{j}$ 's are non-intersecting as no two swap with each other.

We now show that when applying Edelman-Greene insertion to $w$, if $w_{l}$ changes the trajectory of $b_{j}$ at time $l$, then $w_{l}$ will be inserted into the $j$ th column of $P_{n+1-l}(w)$ and each entry bumped during this insertion will in turn insert into the $j$ th column. From this and the definition of Tab, we can conclude that $\operatorname{Tab}(w)=Q(w)$.

If $b_{1}$ has the only non-trivial trajectory amongst the $b_{j}$, then $Q(w)=\operatorname{Tab}(w)$ trivially; there is only one column in $\operatorname{Tab}(w)$. Assume there are multiple $b_{j}$ with nontrivial trajectories. Let $\left\{i_{1}, i_{2}, \ldots, i_{t_{2}}\right\}$ be the trajectory of $b_{2}$. Note $w_{i_{k}}=w_{i_{k+1}}+1$. 
Then, $b_{1}$ has trajectory $\left\{l_{1}, \ldots, l_{t_{1}}\right\}$ with $t_{1} \geq t_{2}$ and $l_{k}>i_{k}$, i.e. the $k$ th from last swap featuring $b_{1}$ comes later than the $k$ th from last featuring $b_{2}$ and so on. Inserting from right to left, we see that upon inserting any $w_{i_{j}}$, we will have already inserted $w_{l_{j}}$. Therefore, $w_{i_{1}}$ will be inserted into the second column as any previously inserted entry will be from the trajectory of $b_{1}$, and thus have inserted into the first column. When $w_{i_{2}}$ is inserted, it too will insert into the second column as $w_{l_{2}}$ will have been inserted into the first column. For identical reasons as before, $w_{i_{1}}$ will remain in the second column upon being bumped. We then see inductively that, unimpeded by other swaps, the trajectory of $b_{2}$ will insert one after another into the second column.

The same argument applies to $b_{3}$ and so on. Thus, $\operatorname{Tab}(w)=Q(w)$.

Corollary 2 For w a Grassmannian word, Edelman-Greene insertion coincides with $R S K$ insertion performed on the reverse word.

Proof The argument in Lemma 5 shows there are no special bumps, so the two algorithms agree.

Remark 1 As pointed out by a referee, Corollary 2 implies that Lemma 5 is essentially a fact about the RSK algorithm, so an alternative approach to proving these two facts is possible. The sketch of this alternative argument is as follows. First, prove the statement of Corollary 2 independently: roughly, Grassmannian words come from 321-avoiding permutations, so special bumps cannot occur. Then, apply Green's theorem [9] to identify the longest increasing subsequences in $w$ with trajectories in the wiring diagram of $W$, proving the statement of Lemma 5.

\subsection{The column reading word}

The only ingredient missing from our argument is a canonical form that is invariant under Little bumps.

Definition 1 For $T$ a Young tableau with columns $C^{1}, C^{2}, \ldots, C^{m}$ where $C^{i}=$ $c_{1}^{i}, c_{2}^{i}, \ldots, c_{k}^{i}$ with $c_{j}^{i}$ being the $(j, i)$ th entry of $T$. We define the column reading word of $T$ to be the word

$$
\tau(T)=C^{m} C^{m-1} \ldots C^{1} .
$$

If $T$ is row and column strict then $P(\tau(T))=T$ and each column of $Q(\tau(T))$ has consecutive entries. For $w$ a reduced word, we define $\tau(w)$ to be $\tau(P(w))$. By the previous observation, $w$ and $\tau(w)$ are Coxeter-Knuth equivalent.

For example, the tableau in Fig. 2 has columns 1245, 36 and 7, so its column word is 7361245 . One can think of the column reading word as closely related to the bottom-up reading word. Since insertion takes place from right to left, the column reading word is in some sense its transpose.

Lemma 6 Let $w$ be a reduced word and $\uparrow$ a Little bump on $w$. Then

$$
Q(\tau(w))=Q(\tau(w) \uparrow)
$$


Proof Let $w$ be a reduced word, $\tau(w)=C^{m} C^{m-1} \ldots C^{1}$ and $\tau(w) \uparrow=D^{m} D^{m-1} \ldots D^{1}$ where $\left|C^{i}\right|=\left|D^{i}\right|$ (note $D^{k}$ is not a priori a column of $P(\tau(w) \uparrow)$ ). Since $\tau(w)$ and $\tau(w) \uparrow$ have the same descent structure, we see $C^{1}$ and $D^{1}$ insert identically. As each entry of $\tau(w) \uparrow$ is decremented at most once and $P(\tau(w))$ is row and column strict, we see

$$
d_{i}^{k} \leq c_{i}^{k} \leq d_{i}^{k}+1 \leq d_{i}^{k+1}
$$

so $d_{i}^{k+1}$ will not bump any $d_{j}^{k}$ with $j \leq i$. Therefore, any entry of $D^{k}$ will stay in the $k$ th column of $P(\tau(w) \uparrow)$ for all $k$, that is the entries of the $k$ th column of $P(\tau(w) \uparrow)$ are $D^{k}$. Thus, $\tau(w) \uparrow$ is a column reading word with identical column sizes, so $Q(\tau(w))=Q(\tau(w) \uparrow)$.

\subsection{Proof of Theorem 1 and its corollaries}

Combining Lemma 6 with Lemmas 3 and 4, we can conclude the following:

Proposition 1 Let $w$ be a reduced word and $\uparrow$ be a Little bump on $w$. Then

$$
Q(w)=Q(w \uparrow)
$$

Proof Let $w$ be a reduced word. There exists a sequence $\alpha_{1}, \alpha_{2}, \ldots, \alpha_{k}$ of CoxeterKnuth moves such that $w=\tau(w) \alpha_{1} \ldots \alpha_{k}$. As $Q(\tau(w))=Q(\tau(w) \uparrow)$ by Lemma 6 , we compute

$$
\begin{aligned}
Q(w) & =Q\left(\tau(w) \alpha_{1} \ldots \alpha_{k}\right) \\
& =Q\left((\tau(w) \uparrow) \alpha_{1} \ldots \alpha_{k}\right) \\
& =Q\left(\left(\tau(w) \alpha_{1} \ldots \alpha_{k}\right) \uparrow\right)=Q(w \uparrow)
\end{aligned}
$$

where the second equality follows from Lemmas 4 and 6 and the third equality follows from Lemma 3.

Proof (Proof of Theorem 1) Let $w$ be a reduced word and $\uparrow_{i_{1}}, \ldots, \uparrow i_{i_{k}}$ be the sequence of canonical Little bumps. By Prop 1 and Lemma 5, we see

$$
Q(w)=Q\left(w \uparrow_{i_{1}} \ldots \uparrow_{i_{k}}\right)=\operatorname{Tab}\left(w \uparrow_{i_{1}} \ldots \uparrow_{i_{k}}\right)=\operatorname{LS}(w) .
$$

We now demonstrate several consequences. First, we show how to use our results to compute the set of $P$-tableaux associated to a given permutation.

For the permutation $\sigma$, let $P(\sigma)=\{P(w): w \in \operatorname{Red}(\sigma)\}$. We outline an algorithm for computing $P(\sigma)$. In order to do so, we must define the Lascoux-Schützenberger tree of a permutation $\sigma[8,11,14]$. This is a tree rooted at $\sigma$; its other nodes are indexed by permutations, defined recursively as follows. If $\sigma$ is Grassmannian, it has no children. Otherwise, the children of $\sigma$ are

$$
\left\{\sigma t_{(r, s)} t_{(i, r)}: i<r \text { and } l\left(\sigma t_{(r, s)} t_{(i, r)}\right)=l(\sigma)\right\}
$$


where $(r, s)$ is the last inversion in $\sigma$ lexicographically. If no such $i$ exists, the children of $\sigma$ are the same as those of $1 \oplus \sigma=1\left(\sigma_{1}+1\right) \ldots\left(\sigma_{n}+1\right)$. As described in [14], the paths from $\sigma$ to each of the leaves encode a canonical seqence of Little bumps which transform any reduced word for $\sigma$ into an associated Grassmannian word.

\section{Algorithm 5 (to compute the Edelman-Greene P-tableau asociated to $\sigma$ )}

1. Compute the Lascoux-Schützenberger tree for $\sigma$.

2. For each leaf of the tree, pick a representative reduced word, e.g. the column word.

3. Apply inverse Little bumps along the tree to each representative until it is a reduced word of $\sigma$. Such bumps are initiated at the swap introducing the inversion $(i, r)$, following the notation used to define the Lascoux-Schützenberger tree.

4. Compute $P(w)$ for each representative. The set of outcomes will be $P(\sigma)$.

In fact, by [18], we can improve efficiency in step 1 slightly by stopping at vexillary (2143-avoiding) permutations, rather than continuing to Grassmannian permutations. Another method for computing $P(\sigma)$ can be found in [15].

Next is a simple consequence of Corollary 1.

Corollary 3 The descent structure of a reduced word $w$ is determined by $Q(w)$.

There is an analogous result for the Robinson-Schensted-Knuth algorithm, which appears first in a paper of Schützenberger [17]. It was subsequently rediscovered by Foulkes [7]. The proof can also be found in the standard reference [19, Lemma 7.23.1]. For sorting networks, this result was proved in [6].

The next is Conjecture 11 from [12], which first appeared as Conjecture 4.3.3 in the appendix of [8].

Corollary 4 Let $w$ be a reduced word and let $\uparrow_{i_{1}}, \uparrow_{i_{2}}, \ldots, \uparrow_{i_{k}}$ be any sequence of Little bumps such that

$$
v=w \uparrow i_{1} \ldots \uparrow i_{k}
$$

is a Grassmannian word. Then Tab $(v)=L S(w)$.

This follows from Prop 1. We can extend this result further. Let $\lambda$ be a partition with $w$ a Grassmannian word whose corresponding tableau is of shape $\lambda$. The permutation $\sigma$ associated to $w$ can be characterized by the number of initial fixed points. A Grassmannian permutation is minimal if it has no initial fixed points. Note the minimal Grassmannian permutation of a given shape is unique in $S_{\infty}$. Recall two reduced words communicate if there exists a sequence of Little bumps and inverse Little bumps changing one to the other.

We next provide proofs of our remaining main theorems (of Lam's conjecture, and of the dual equivalence graph structure of the set of Coxeter-Knuth moves).

Proof (of Theorem 2) Let $v$ and $w$ be reduced words. Suppose first that $v$ and $w$ communicate. Then by Theorem 1 , we have that $Q(v)=Q(w)$.

Conversely, suppose that $Q(v)=Q(w)$. By applying the canonical sequence of Little bumps, $w$ can be changed to the Grassmannian word $w^{\prime}$ and $v$ to the Grassmannian 


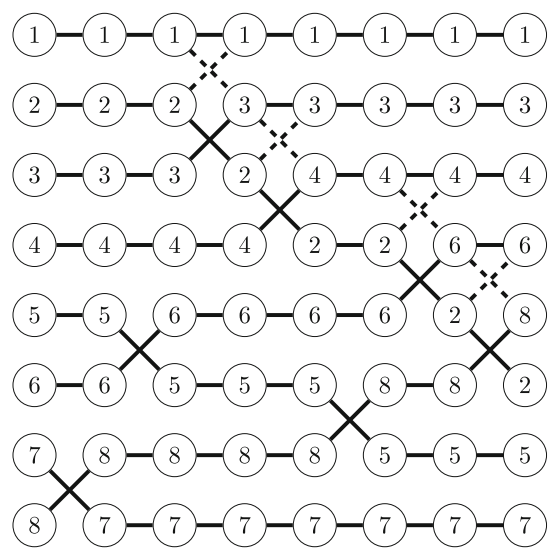

Wiring diagram for $w$

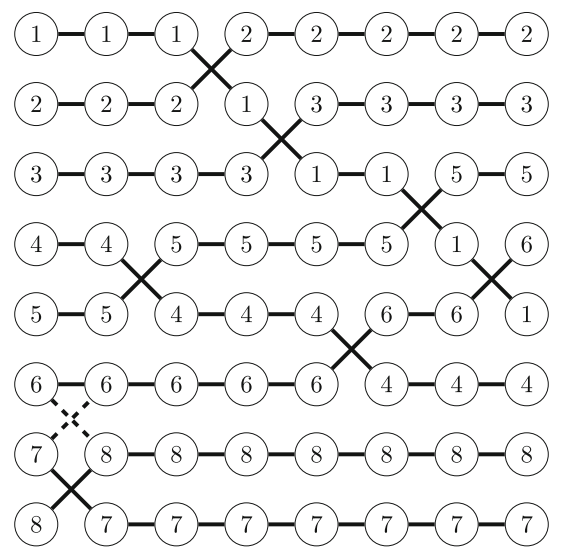

Wiring diagram for $w \uparrow_{7} \uparrow_{5}$

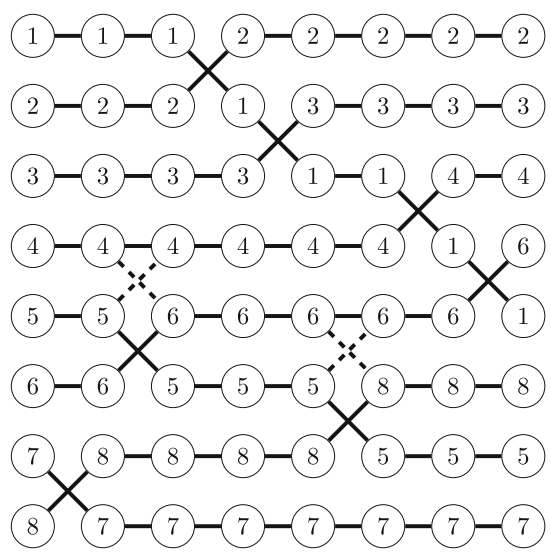

Wiring diagram for $w \uparrow 7$

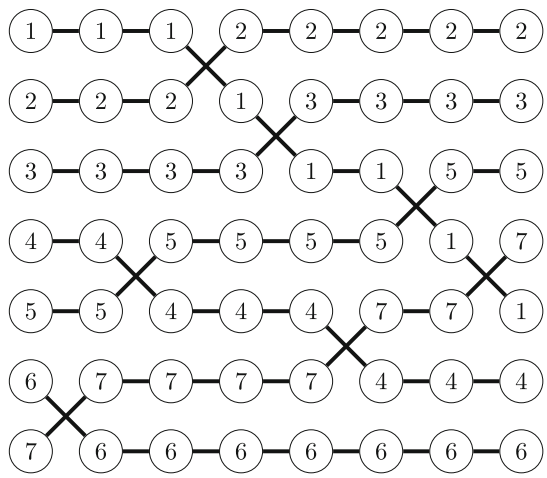

Wiring diagram for $w \uparrow_{7} \uparrow_{5} \uparrow_{1}$

Fig. 6 Removing a fixed point from the Grassmannian word $w=7523645$ via the canonical sequence of bumps

word $v^{\prime}$. Since Little bumps are invertible, $Q(w)=Q\left(w^{\prime}\right)$ and $Q(v)=Q\left(v^{\prime}\right)$, we can conclude that $v$ and $w$ communicate if Grassmannian permutations of the same shape communicate. To show this, we demonstrate a sequence of Little bumps that will remove a fixed point at the beginning of an arbitrary Grassmannian permutation. Let $\sigma=a_{1} \ldots a_{k} b_{1} \ldots b_{n-k}$ be a Grassmannian permutation with $a_{k} b_{1}$ its sole descent. Our sequence is constructed by initiating a Little bump at the last swap featuring each $b_{j}$, beginning with $b_{1}$. See Fig. 6 for an example. If $\sigma$ has initial fixed points, this sequence will decrement each entry of $w$, removing an initial fixed point.

We now verify that our sequence works as described. First, we must verify that the swap locations at which we begin a Little bump are valid choices, that is that removing that swap from $w$ leaves a reduced word. To see this, note that the first such swap chosen is the swap between $a_{k}$ and $b_{1}$, the last swap in $w$. This bump will decrement every entry in the trajectory of $b_{1}$. After the first Little bump, the second 
swap chosen is the last in the trajectory of $b_{2}$. Since the trajectories of all $b_{j}$ with $j>2$ are unaffected by the initial Little bump, this is the last swap for both $b_{2}$ and $a_{k}$, so removing it leaves a reduced word. This bump will decrement every entry in the trajectory of $b_{2}$. Note because we have already decremented the swaps in the trajectory of $b_{1}$ and these trajectories were initially disjoint, they will remain disjoint after the second Little bump. Applying this line of reasoning inductively, we see that each Little bump in the sequence is a valid Little bump which decrements every entry of each trajectory. We have now shown $v$ and $w$ communicate if $Q(v)=Q(w)$.

Therefore, any Grassmannian permutation communicates with the minimal permutation of that shape. From this, we can conclude any two Grassmannian permutations with the same shape communicate.

For a permutation $\sigma$, we define the graph $G_{\sigma}=(V, E)$ where $V=\operatorname{Red}(\sigma)$ and $(v, w) \in E$ if $v=w \alpha$ where $\alpha$ is a Coxeter-Knuth move. In [4], the notion of a dual equivalence graph is introduced and axiomatized. Such graphs satisfy certain connectivity properties and can be assigned weights in a specific fashion. The axioms are quite complicated, and we refer the reader to [16] or [4] for a complete characterization. The existence of such graphs imply Schur positivity for associated symmetric functions. It is known that the graphs on words induced by Knuth equivalence and dual Knuth equivalence, respectively, satisfy these axioms [16, Theorem 3.2]. Additionally, we make use of the fact that the disjoint union of dual equivalence graphs with comparable labels is a dual equivalence graph.

Proof (of Theorem 3) Our strategy here follows an original proof of this idea due to Sara Billey (private communication). We first examine the case where $\sigma$ is a Grassmannian permutation. By Corollary 2, RSK and Edelman-Greene insertion coincide on $\operatorname{Red}(\sigma)$. Since Knuth transformations coincide with Coxeter-Knuth transformations, we see the $G_{\sigma}$ is a dual equivalence graph as it is the same as the graph on $\operatorname{Red}(\sigma)$ induced by Knuth transformations.

We now study the image of $G_{\sigma}$ under Little bumps. By Lemma 3, we see $G_{\sigma} \cong$ $\left(G_{\sigma}\right) \uparrow$, the image of $G_{\sigma}$ under any Little bump. Therefore, for an arbitrary permutation $\sigma$, we see $G_{\sigma} \cong G_{\sigma_{1}} \sqcup \cdots \sqcup G_{\sigma_{k}}$ where $\sigma_{1}, \ldots, \sigma_{k}$ are Grassmannian permutations. Since the disjoint union of dual equivalence graphs is a dual equivalence graphs, we see $G_{\sigma}$ is a dual equivalence graph.

Finally, we show how to embed Robinson-Schensted insertion and RSK in the Little map. In doing so, we recover a modified version of [12, Theorem 9] through a much simplified argument. This embedding was first predicted as Conjecture 4.3.1 in the appendix of [8].

Theorem 6 Let $\sigma=\sigma_{1} \ldots \sigma_{n} \in S_{n}$, so that $w(\sigma)=\left(2 \sigma_{n}-1\right) \ldots\left(2 \sigma_{1}-1\right)$ is a reduced word as it has no repeated entries. Let $R S(\sigma)=\left(P^{\prime}(\sigma), Q^{\prime}(\sigma)\right)$ be the output of Robinson-Schensted insertion applied to $\sigma$. Upon applying the transformation $k \mapsto(k+1) / 2$ to the entries of $L S(w(\sigma))$, we obtain $Q^{\prime}(\sigma)$. We can obtain $P^{\prime}(\sigma)$ by applying the same transformation to $\operatorname{LS}\left(w\left(\sigma^{-1}\right)\right)$.

Proof Since LS $(w)=Q(w)$ and there are no special bumps, Edelman-Greene insertion will perform the same insertion process on $w$ as Robinson-Schensted insertion 
performs on $\sigma$. Therefore, upon applying the transformation $k \mapsto(k+1) / 2$, we see $\operatorname{LS}(w(\sigma))=Q(w(\sigma))=Q^{\prime}(\sigma)$. Since $\operatorname{RS}\left(\sigma^{-1}\right)=\left(Q^{\prime}(\sigma), P^{\prime}(\sigma)\right)$ (see e.g. [19]), we can obtain $P^{\prime}(\sigma)$ by applying the same transformation to $\operatorname{LS}\left(w\left(\sigma^{-1}\right)\right)$.

Since RSK can be embedded in Robinson-Schensted insertion (see Sect. 7 of [12] for a description of this process), Theorem 6 recovers an embedding of RSK into the Little map as well.

Acknowledgments We would like to thank Omar Angel, Sara Billey, Vadim Gorin, Ander Holroyd, Thomas Lam, David Little, Eric Nordenstam, Dan Romik, Balint Virag and Peter Winkler for helpful discussions and the referees for helpful comments. This research began while we were visiting the Mathematical Sciences Research Institute in 2012, for the program in Random Spatial Processes. This project made heavy use of computer experiments in Sage [20]. Also, David Little's applet [13] was very helpful in understanding the Little bijection.

\section{References}

1. Angel, O., Gorin, V., Holroyd, A.E.: A pattern theorem for random sorting networks. arXiv, preprint. arXiv:1110.0160. (2011)

2. Angel, O., Holroyd, A.E.: Random subnetworks of random sorting networks. Electron. J. Comb. 17(23), $1(2010)$

3. Angel, O., Holroyd, A.E., Romik, D., Virág, B.: Random sorting networks. Adv. Math. 215(2), 839-868 (2007)

4. Assaf, Sami H,: Dual equivalence graphs I: a combinatorial proof of LLT and macdonald positivity. arXiv, preprint. arXiv:1005.3759. (2010)

5. Chhita, S., Johansson, K., Young, B.: Asymptotic domino statistics in the Aztec diamond. arXiv, preprint. arXiv:1212.5414. (2012)

6. Edelman, P., Greene, C.: Balanced tableaux. Adv. Math. 63(1), 42-99 (1987)

7. Foulkes, H.O.: Enumeration of permutations with prescribed up-down and inversion sequences. Discret. Math. 15(3), 235-252 (1976)

8. Garsia, Adriano.: The saga of reduced factorizations of elements of the symmetric group. Laboratoire de combinatoire et d'informatique mathématique. Université du Québec à Montréal, Montréal, Canada (2002)

9. Greene, Curtis: An extension of schensted's theorem. Adv. Math. 14(2), 254-265 (1974)

10. Lam, T.: Stanley symmetric functions and Peterson algebras. Arxiv, preprint arXiv:1007.2871, (2010)

11. Lascoux, A., Schützenberger, M.P.: Schubert polynomials and the Littlewood-Richardson rule. Lett. Math. Phys. 10(2), 111-124 (1985)

12. Little, David P.: Factorization of the Robinson-Schensted-Knuth correspondence. J. Comb. Theory Ser. A 110(1), 147-168 (2005)

13. Little, David P.: Diagram Viewer: a java applet implementing the Little bijection. http://www.math. psu.edu/dlittle/DiagramViewer.zip, (2012)

14. Little, D.P.: Combinatorial aspects of the Lascoux-Schützenberger tree. Adv. Math. 174(2), 236-253 (2003)

15. Reiner, Victor, Shimozono, Mark: Plactification. J. Algebr. Comb. 4(4), 331-351 (1995)

16. Roberts, Austin.: Dual equivalence graphs revisited and the explicit Schur expansion of a family of LLT polynomials. J. Algebr. Comb. 39(2), 1-40 (2013)

17. Schützenberger, M.P.: Quelques remarques sur une Construction de Schensted. Math. Scand 12, 117128 (1963)

18. Stanley, R.P.: On the number of reduced decompositions of elements of Coxeter groups. Eur. J. Comb. 5, 359-372 (1984)

19. Stanley, R.P.: Enumerative Combinatorics, vol. 2. Cambridge Univ Pr, Cambridge, MA (2001)

20. Stein, W.A. et al.: Sage Mathematics Software (Version 5.3). The sage development team. http://www. sagemath.org. (2012) 